\title{
MOMENTS OF ANALYTIC FUNCTIONS
}

R. P. BOAS, JR.

There are many theorems which state that an analytic function which is of sufficiently slow growth in a half-plane and tends sufficiently rapidly to zero along an interior line must vanish identically. Recently a theorem of this sort was proved by San Juan [4] and Sunyer Balaguer [5], where the condition of rapid approach to zero is expressed indirectly by the smallness of a set of moments. In the more precise formulation of Sunyer Balaguer, the theorem is as follows.

THEOREM 1. If $f(z)$ is regular and bounded for $x \geqq 0$, and

$$
\int_{0}^{\infty}|f(x)| x^{n} d x<\Gamma(\beta n+1), \quad \beta<1,
$$

for an infinity of $n$, then $f(z) \equiv 0$.

This theorem, in a still more general form, can be deduced from a theorem of Ahlfors and Heins $[1 ; 3]$ on subharmonic functions. Stated in the form appropriate for analytic functions of exponential type, this reads as follows.

THEOREM 2. If $f(z)$ is regular and of exponential type for $x \geqq 0$, bounded on the imaginary axis, and not identically zero, then for some number $c$ we have $\lim _{r \rightarrow \infty} r^{-1} \log \left|f\left(r e^{i \theta}\right)\right|=c \cos \theta$, for all $\theta$ in $(-\pi / 2$, $\pi / 2$ ) except a set of outer capacity 0 , and for each $\theta$ in this interval if $r$ is excluded from a set of finite logarithmic length.

A function $f(z)$ is of exponential type if $|f(z)| \leqq A e^{k|z|}$ for some $k$ and $A$; the logarithmic length of $E$ is $\int_{E} x^{-1} d x$.

I use the second part of Theorem 2 to prove the following stronger form of Theorem 1.

THEOREM 3. If $f(z)$ is regular and of exponential type for $x \geqq 0$, and is bounded on the imaginary axis, and if

$$
\int_{0}^{\infty}\left|f\left(r e^{i \theta}\right)\right| r^{n} d r<n^{n} e^{-n \phi(n)}, \quad \phi(n) \rightarrow \infty,
$$

for some $\theta,-\pi / 2<\theta<\pi / 2$, and for an infinity of $n$, then $f(z) \equiv 0$.

Theorem 1 is effectively the case in which $\phi(n)=(1-\beta) \log n$.

Presented to the Society, September 3, 1954; received by the editors May 28, 1954 and, in revised form, August 30, 1954. 
We may suppose, without loss of generality, that $\phi(n)<\log n$. Let $\alpha(n)$ be a function such that $0<\alpha(n) \uparrow 1$, and $\{1-\alpha(n)\} \log n \rightarrow \infty$, but is $o\{\phi(n)\}$. Let $\mu(E)$ denote the logarithmic length of $E$, and suppose that $\left|f\left(r e^{i \theta}\right)\right|>e^{-n}$ on a set $E_{n}$ in $\left(n^{\alpha(n)}, \lambda n^{\alpha(n)}\right), \lambda>1$, where $n$ is an integer for which (1) holds. We have

and hence

$$
\begin{aligned}
n^{n} e^{-n \phi(n)} & >\int_{E_{n}}\left|f\left(r e^{i \theta}\right)\right| r^{n} d r>e^{-n} \int_{E_{n}} r^{n+1} r^{-1} d r \\
& >e^{-n} n^{(n+1) \alpha(n)} \mu\left(E_{n}\right)
\end{aligned}
$$

$$
\begin{aligned}
\mu\left(E_{n}\right) & <\exp \{n+n \log n-(n+1) \log n+(n+1) o[\phi(n)]-n \phi(n)\} \\
& =o(1) .
\end{aligned}
$$

Thus $\left|f\left(r e^{i \theta}\right)\right| \leqq e^{-n}$ on $\left(n^{\alpha(n)}, \lambda n^{\alpha(n)}\right)$ except at most for a set whose logarithmic length approaches zero as $n \rightarrow \infty$ through the values satisfying (1). In other words, in the specified intervals, except for a set of infinitesimal logarithmic length,

$$
\begin{aligned}
r^{-1} \log \left|f\left(r e^{i \theta}\right)\right| & \leqq-n / r \leqq-n / n^{\alpha(n)}=-\exp \{[1-\alpha(n)] \log n\} \\
& \rightarrow-\infty .
\end{aligned}
$$

Since the logarithmic length of $\left(n^{\alpha(n)}, \lambda n^{\alpha(n)}\right)$ is $\log \lambda$, we have $r^{-1} \log \left|f\left(r e^{i \theta}\right)\right| \rightarrow-\infty$ on a set of intervals of infinite logarithmic length. By Theorem 2, this can happen only if $f(z) \equiv 0$.

It is not essential to suppose that $f(i y)$ is bounded, since Theorem 2 remains true if we assume only, for example, that

$$
\int_{-\infty}^{\infty}\left(1+y^{2}\right)^{-1} \log ^{+}|f(i y)| d y<\infty
$$

(see [2]); for the application to Theorem 3 we need still less, for example that $\int_{-\infty}^{\infty} y^{-2} \log |f(i y) f(-i y)| d y<O(1)$.

\section{REFERENCES}

1. L. Ahlfors and M. Heins, Questions of regularity connected with the PhragmenLindelöf principle, Ann. of Math. (2) vol. 50 (1949) pp. 341-346.

2. R. P. Boas, Jr., Asymptotic properties of functions of exponential type, Duke Math. J. vol. 20 (1953) pp. 433-448.

3. J. Lelong-Ferrand, Étude au voisinage de la frontière des fonctions surharmoniques positives dans un demi-espace, Ann. Ecole Norm. (3) vol. 66 (1949) pp. 125-159.

4. R. San Juan, L'accroissement des moments d'une fonction holomorphe dans un angle, C. R. Acad. Sci. Paris vol. 236 (1953) pp. 1941-1943.

5. F. Sunyer Balaguer, Sobre los momentos de las funciones holomorfas y acotadas en un angulo, Revista Matemática Hispano-Americana (4) vol. 13 (1953) pp. 241246.

NORTHWESTERN UNIVERSITY 